\title{
Sporadic Abdominal Wall Desmoid: Is it Time to Change Our First-Line Approach?
}

\author{
Sylvie Bonvalot, MD, PhD \\ Department of Surgical Oncology, Gustave Roussy, Villejuif, France
}

The team of the Royal Marsden of London reported a series of 50 patients who underwent the resection of a primary sporadic abdominal wall desmoid tumor (DT). ${ }^{1}$ This field is changing rapidly as new data accumulates, and a personalized approach that takes into account the initial tumor size and/or the evolution after initial surveillance is becoming the preferred method. ${ }^{2}$

This series confirms recent data $^{3}$ indicating the strong predominance $(96 \%)$ of females, particularly those at reproductive age, with tumors in this location. During the study period, the authors systematically performed surgical resection following a biopsy. This approach resulted in an abdominal wall defect that required a prosthetic mesh in $94 \%$ of patients. Not surprisingly, prolonged postoperative pain, hernia or infection affected approximately one patient out of five. Interestingly, no obstetrical impairment due to the mesh repair was observed among the ten pregnancies that reached full term. This study confirms the reports of others ${ }^{3,4}$ that the prognosis is good for abdominal wall DT compared with extra-abdominal DT. In other words, excellent local control was achieved with surgery alone, with low but significant morbidity. Combining all studies that examined the resection of abdominal wall DT, the estimated recurrence rate after surgery calculated by the authors is approximately $5 \%$.

The current issue is when and for whom should we propose this efficient surgical resection? The authors acknowledge that their strategy has evolved recently to adopt active surveillance as a first-line therapy, as better knowledge of the disease has been achieved through the

(C) Society of Surgical Oncology 2014

First Received: 24 March 2014;

Published Online: 9 April 2014

S. Bonvalot, MD, PhD

e-mail: sylvie.bonvalot@gustaveroussy.fr most recent analysis. Indeed, a recent study ${ }^{3}$ reported on 147 patients with abdominal wall desmoid, 102 of whom underwent an initial observation. Approximately one-third of the patients remained stable and one-third exhibited spontaneous regression. Only $16 \%$ of the patients received operations at 3 years because of pain or progression. The vast majority of progressions occur during the first 3 years after diagnosis. Adopting a 'wait and see' approach enables the identification of patients who really require treatment, the best of which is surgery in this location, as shown in this paper. It is likely that abdominal wall desmoids were overtreated in the past, and surgical resection should now only be proposed for selected cases.

The risk of a 'wait and see' approach is that the progression may create the need for a more extensive operation, and this risk should be weighed against the risk of performing an unnecessary surgery in the majority of patients. Therefore, the main issue is to detect the few patients who are at a higher risk of progression. In this paper, a tumor size greater than $7 \mathrm{~cm}$ was the only factor that affected disease-free survival. This threshold was also found in previous papers. ${ }^{3,4}$ Moreover, when we observe significant percentages of progression according to RECIST (Response Evaluation Criteria In Solid Tumors), the baseline tumor size is important to consider. Others factors, such as the location relative to the inguinal ligament and patient compliance to strict initial monitoring should also be taken into consideration. In the event of progression, the best salvage treatment in this location is clearly surgery. Whether medical treatment can be interposed as a secondline before deciding to resort to surgery is under investigation; patients who develop a DT just after pregnancy are likely the best candidates for this approach. The risk of progression for an in situ DT during pregnancy was evaluated recently in a small series (29 patients) and found to be approximately $50 \%$, but the condition was safely managed in the majority of cases..$^{5}$ We need further 
collaborative studies on this specific situation to properly advise patients and adapt the follow-up.

Because DT exhibits an unpredictable clinical course despite having an indistinguishable morphology, translational research is crucial to identify the predictive factors of progression in addition to the clinical parameters. A specific S45-F mutation of $\beta$-catenin indicated a worse recurrencefree survival in a series of operated patients. ${ }^{6}$ Interestingly, the percentage of S45F-mutated DT increases with increasing tumor size ${ }^{6}$ and is more prevalent in extra-abdominal sites (which also receive a worse prognosis). However, somatic markers are not the only factors to consider because the tumor may completely shrink spontaneously and undergo changes in size (similar to an accordion) according to external events (pregnancy, trauma, surgery performed at another site, among other events). Certainly, the host environment is also involved, and immunotherapy could be tested in the future in the worse cases, as in other malignancies. Additionally, the observation of a higher frequency of sporadic colon cancers in a family of patients with DT compared with the general population raises the question of polymorphisms. ${ }^{3}$ The impact of this past history on individual progression is also being evaluated prospectively (ClinicalTrials.gov identifier NCT01801176).

This study reveals that teams with sarcoma expertise, who were previously convinced of the merit of front-line surgery, are now moving toward adopting a more conservative approach for the first-line treatment of abdominal wall DT. The good results of surgery demonstrated by the present study support it as a safe salvage treatment when surveillance fails. Biological markers are urgently needed to individualize treatment strategy from the beginning.

\section{REFERENCES}

1. Wilkinson MJ, Chan KE, Hayes AJ, Strauss DC. Surgical outcomes following resection for sporadic abdominal wall fibromatosis. Ann Surg Oncol. 2014 (in press).

2. Gronchi A, Colombo C, Le Péchoux C, et al. Sporadic desmoidtype fibromatosis: a stepwise approach to a non-metastasising neoplasm - a position paper from the Italian and the French Sarcoma Group. Ann Oncol. 2014;25(3):578-83.

3. Bonvalot $\mathrm{S}$, Ternès $\mathrm{N}$, Fiore $\mathrm{M}$, et al. Spontaneous regression of primary abdominal wall desmoid tumors: more common than previously thought. Ann Surg Oncol. 2013;20(13):4096-102.

4. Salas S, Dufresne A, Bui B, et al. Prognostic factors influencing progression-free survival determined from a series of sporadic desmoid tumors: a wait-and-see policy according to tumor presentation. J Clin Oncol. 2011; 29(26):3553-58.

5. Fiore M, Coppola S, Cannell AJ, et al. Desmoid-type fibromatosis and pregnancy: a multi-institutional analysis of recurrence and obstetric risk. Ann Surg 2013 (in press).

6. Colombo C, Miceli R, Lazar AJ, et al. CTNNB1 45F mutation is a molecular prognosticator of increased postoperative primary desmoid tumor recurrence: an independent, multicenter validation study. Cancer. 2013;119(20):3696-702. 